\title{
DISPERSION COMPENSATION IN OPTICAL COMMUNICATION LINK USING APF'S
}

\author{
Sandeep Singh \\ Vidya College of \\ Engineering, Meerut, \\ U.P., India-250005
}

\author{
Vaibhav Bhushan Tyagi \\ Vidya College of \\ Engineering, Meerut, \\ U.P., India-250005
}

\author{
Anuj Bhardwaj \\ Hindustan Institute Of \\ Technology \& \\ Management, \\ Agra, U.P, India-282007
}

\author{
Neeraj Gupta \\ Anand Engineering \\ College, Agra, \\ U.P, India-282007
}

\begin{abstract}
All pass filters (APFs) are used in dispersion compensation which is the foremost requirement in an optical fiber link. All pass filters can correct any order of dispersion by the careful design of multistage all pass filters starting from very simple components with the use of $\mathrm{N}$ port devices. Multiple channels, as in wavelength division multiplexed (WDM) system, can be compensated with a single device since these filters are periodic in phase response. In this paper we have designed and implemented these filters to compensate dispersion an some results has shown.
\end{abstract}

Keywords: Optical communication, optical fibers, wavelength division multiplexed systems, dispersion compensation all pass filters.

\section{INTRODUCTION}

All Pass filters are used to compensate the chromatic dispersion in wavelength division multiplexed (WDM) optical fiber communication system [1]. Optical fiber communication is a way of transmitting the information from one place to another by modulating the light signal with the information signal. The light signal required for communication is generated using the spontaneous and stimulated emission occurring in light emitting diodes (LEDs) and LASERs [2]. Since the energy levels are not discrete so mono-chromaticity of the light signal is lost and it introduces chromatic dispersion. The number of compensating techniques has been reported in the literature [3], [4], [5], [6] including dispersion compensating fibers (DCFs), Fiber Bragg gratings (FBGs), Electronic Dispersion compensation (EDC) each having its own advantages and disadvantages. In WDM system where a number of frequencies are interleaved, dispersion is compensated using all pass filters [7]. All pass filters are linear systems having variable phase response and constant amplitude response. The variable phase response of the APFs makes them to be used as the phase equalizers to compensate the chromatic dispersion. The need of dispersion compensation, general properties of all pass filters, the design and implementation of all pass filters along with tunable dispersion compensation all pass filters have been discussed in this paper.

\section{NEED}

Due to the presence of chromatic dispersion the light pulse carrying the required information is spread into various components and each component travel differently along the optical fiber with different velocity and hence reach at the receiver at different times which distorts the information and can't be interpreted in the correct manner This is called group velocity dispersion (GVD) which cause the light pulses to spread in fibers, degrading signals over long distances [8-11]. In order to remove the spreading of the optical or light pulses, the dispersion compensation is the most key feature required in optical fiber communication system.

The traditional techniques like DCFs, FBGs, and EDC are not suitable for dispersion compensation in WDM system. DCFs give high insertion loss, large footprint, and nonlinear distortions when the input signal is high etc. Also for the multiple channels in WDM system, the number of DCFs has to be installed making the system complex and costly. The same problem is with the FBGs which compensate the dispersion by the recompression of an optical signal. For different frequencies different architectures of the FBGs have to be introduced along the fiber link. EDC is rendered ineffective for WDM system since it is complex and also not a direct method of compensation as it involve the optical to electronic and electrical to optical conversions making the WDM communication slow which can't be tolerated in this growing world hence the need of all pass filters is realized by which the multiple channels can be compensated with a single device because of the periodic properties of the phase response of these filters [12-15].

\section{ALL PASS FILTERS (APF)}

The dispersion compensation using digital filters is a new technique for the removal of phase distortions of an optical signal. After the various channels have been multiplexed by the wavelength interleaver over the single fiber the next step is to compensate the phase distortions due to different group delays for different channels [1], [7]. Dispersion compensating fibers [2], [3] (with opposite chromatic dispersion as that of channels) are not used these days as 
they introduce large footprint, high insertion loss, introduce nonlinear distortion etc, hence they have been replaced by all pass filter structure. It is a special filter with flat magnitude spectrum and non-linear phase spectrum, so it compensates phase distortion without affecting magnitude spectrum of signals [8], [9]. These all pass filters (APF's) are linear systems, which have an amplitude response that is constant over all frequencies and a phase response that varies with frequency. The period of frequency response of all pass filters is usually referred to as free spectral range (FSR). Mathematically, the frequency response of a filter is written as

$H(\omega)=|H(\omega)| \exp [j \Phi(\omega)]$

then for an APF $|\mathrm{H}(\omega)|=\mathrm{c}$ where $\mathrm{c}$ is a constant and $\Phi(\omega)$ can be made arbitrarily close to any desired phase response. With this characteristic the nth-order dispersion is evaluated as 1/ FSRn, further group delay can be enhanced by adding more number of stages [12]. However it increases loss in the system. Adding stages to the APF help in recovering group delay that is lost when the FSR is increased [12], [13].

The dispersion compensation obtained experimentally is $\mathrm{D} \sim \mathrm{N} / \mathrm{FSR}^{2} \Delta^{2}$

(2)

Where $\mathrm{N}$ is number of channels and $\Delta$ is distance of poles and zeros of the unit circle. The dispersion may be increased by reducing the FSR with the introduction of more number of stages or by reducing the $\Delta$.

\section{APF DESIGN IMPLEMENTATION}

For the design of an APF, a four port device with frequency independent matrix elements can be considered. By connecting any one of the outputs through a delay to any one of the inputs a single stage APF can be realized [12]. APF may be implemented using Directional couplers, Mach-Zehnder interferometer, and thin film filter as shown below in Fig.1-4:

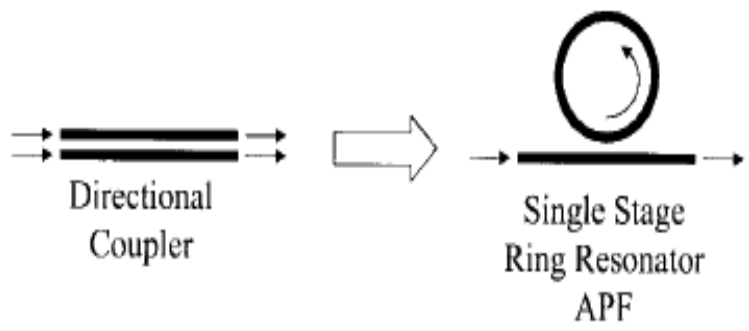

Fig 1. Single stage APF using directional coupler [12].

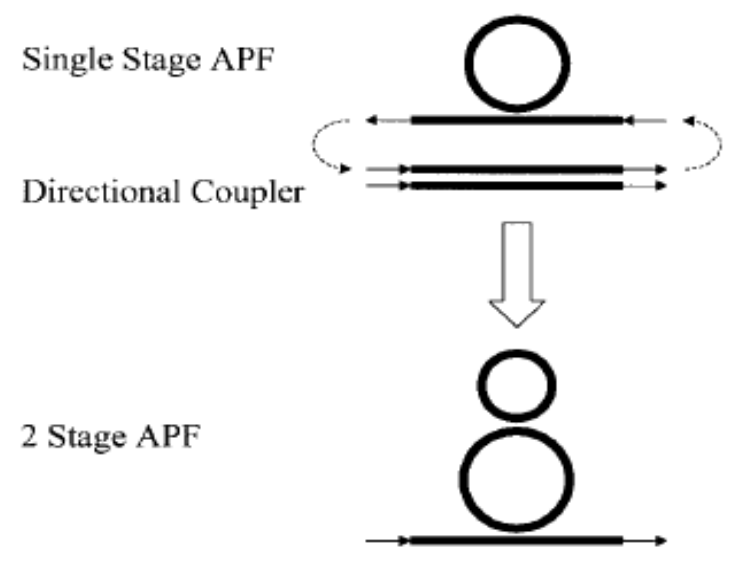

Fig 2. Two stage APF [12].

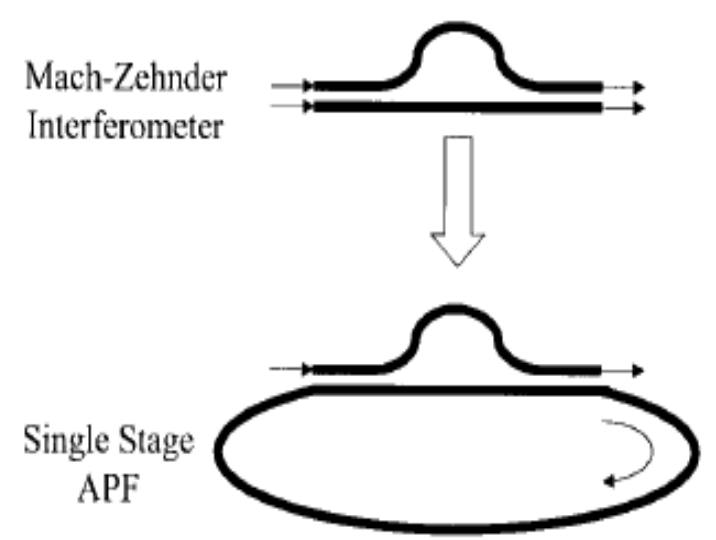

Fig 3.Single stage APF using Mach-Zehnder interferometer [12]

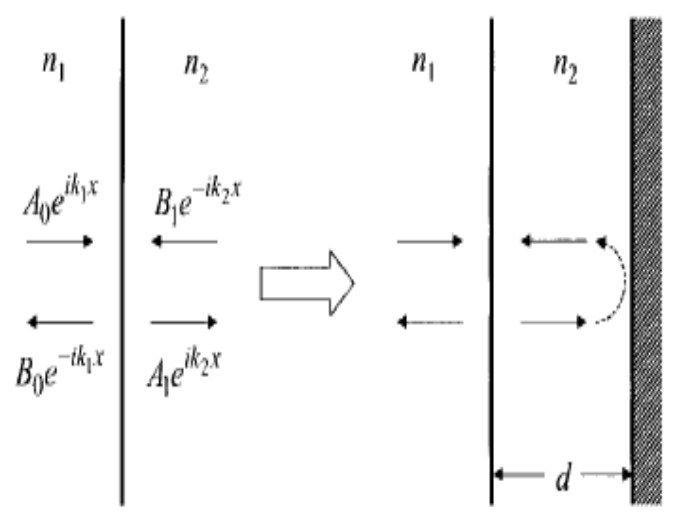

(a)

(b)

Fig 4. Thin-film example: (a) Interface between two dielectrics. The scattering matrix relates the "input" amplitudes A0 and B1 to the "output" amplitudes A1 and B0. (b) By connecting the "output" A1 to the "input" B1 through a delay (using a 100\% reflector a distance $\mathrm{d}$ away), a single-stage APF is obtained. This is exactly the familiar Gires-Tournois interferometer [12]. 


\section{TUNABLE DISPERSION COMPENSATION ALL PASS FILTERS}

Chromatic dispersion compensation is critical for high bit rate light wave systems. Reconfigurable optical networks introduce a need for tunable dispersion compensation since different routes may have different cumulative dispersions [14]. In addition, tunable dispersion compensation is required for high bit rate nonlinear systems whose optimal dispersion depends on the channel power which may fluctuate over time. Different wavelengths have different cumulative dispersions at the receiver, and a device capable of applying varying amounts of dispersion compensation to each channel is needed. Because of the large number of channels in dense wavelength-division-multiplexed (WDM) systems, periodic filters are advantageous compared to single channel devices which require a unique filter for every WDM channel [14], [15]. Tunable dispersion compensation filters are of two types:

\subsection{MEMS Compensation All Pass Filters}

The tunable all-pass filter is based on the mechanical antireflection switch (MARS) device, which is a variablethickness Fabry-Perot cavity consisting of a silicon substrate, an air gap, and a quarter-wave thick dielectric membrane. A silicon nitride layer is used for the membrane, and the gap is nominally $3 \lambda \mathrm{o} / 4$. The cavity formed by the membrane and top surface of the substrate yields a reflection of about $70 \%$. The gap is varied from $3 \lambda$ $\mathrm{o} / 4$ to $\lambda \mathrm{o} / 4$ by applying a voltage to electrodes on top of the membrane as shown in Fig.5.
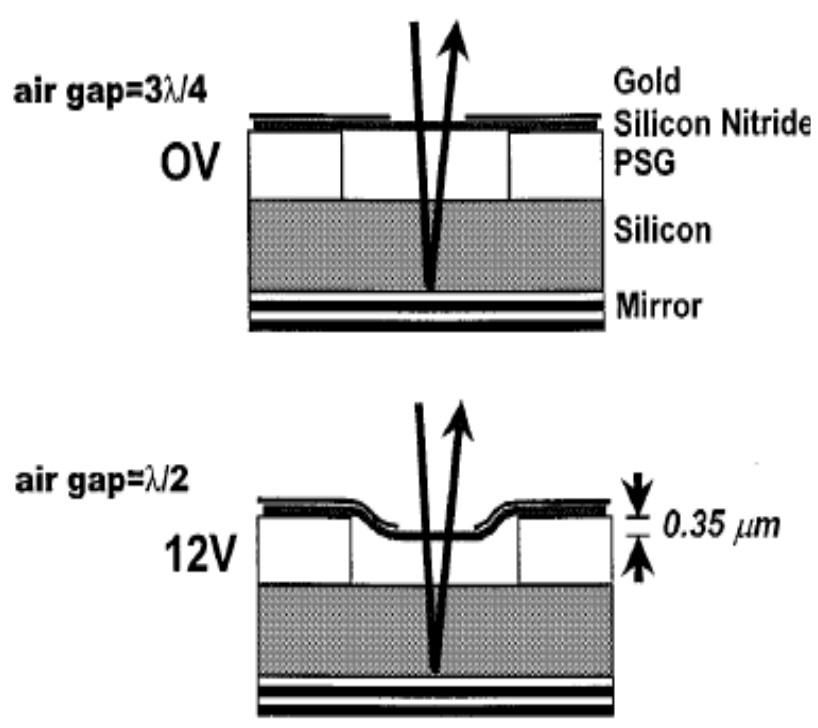

Fig 5. MEMS all-pass filter schematic showing the change in air gap with applied voltage [14].

The voltage creates an electrostatic force that pulls the membrane closer to the substrate surface, while the membrane tension provides a linear restoring force. At a gap of $\lambda \mathrm{o} / 4$, the reflection is reduced to $\sim 0 \%$ since the silicon nitride acts as an antireflection coating for the silicon substrate. To make an all-pass filter, the aim is to use Fabry-Perot cavity as a tunable, partial reflector and add a high reflectance coating to the back side of the substrate [14]. A reflectivity $>97 \%$ is obtained using a multi-layer stack. The substrate thickness L determines the free spectral range FSR $=\mathrm{c} / 2 \mathrm{ngL}$, where ng is the group index. For a 100-GHz FSR, the silicon thickness is $411 \mathrm{~m}$. By selecting the filter period equal the channel spacing in a WDM system, multiple channels can be compensated. The filter dispersion is $\mathrm{D}=\mathrm{d} \tau / \mathrm{d} \lambda(\mathrm{ps} / \mathrm{nm})$. For a completely tunable all-pass filter, both the partial reflector and the cavity optical length must be tunable. By varying the applied voltage, the partial reflectance of the front mirror is changed. For tuning $\Phi \mathrm{n}$, the substrate is mounted on a thermo-electric cooler, and the cavity optical thickness is tuned via the thermooptic effect. Tuning of the cavity length can also be used to compensate for variations in the fabricated cavity length from the design nominal [14].

\subsection{Integrated All Pass Filters for Tunable Dispersion Compensation}

Two parameters control its group delay response, the phase Фand power coupling ratio kr. By using a multistage filter where the parameters are chosen optimally for each stage, a constant dispersion (or any desired response) can be approximated over a large portion of the FSR, thus yielding a large bandwidth utilization factor [15]. It is critical to achieve the design values for these parameters, and fabrication-induced variations on the coupling ratios must be minimized. The new all-pass filter architectures are shown in Fig.6 (a), (b) and (c).

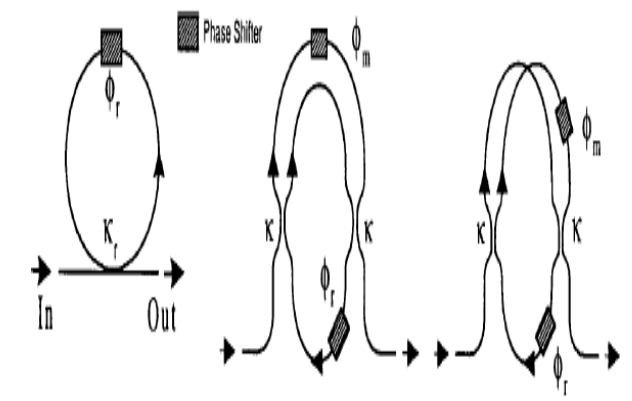

Fig 6. (a) Ring resonator all-pass filter with a fixed coupling ratio, and fully tunable ring

resonator all-pass filters with (b) an asymmetric MZI and (c) a symmetric MZI [15].

The single coupler is replaced with a Mach-Zehnder interferometer (MZI). The MZI is curved to minimize any increase in the feedback path length. The advantage is that a phase shifter can be used to tune the effective coupling ke into the feedback path, thus a completely tunable all-pass filter is easily realized with two phase shifters, one to set ke and one to tune the resonant wavelength. The tolerances on the couplers k composing the MZI are substantially relaxed compared to the tolerance on kr. In Fig. (b), the MZI path lengths are different by a length $\Delta \mathrm{L}=\pi$ dsep where dsep is the separation of the MZI arms. The effective coupling is given by ke which can be set to zero at a given wavelength by choosing $\Phi \mathrm{m}$ appropriately [15]. 
$\mathrm{k}_{\mathrm{e}}=4 \mathrm{k}(1-\mathrm{k}) \cos ^{2}\left(\left[2 \pi \mathrm{n}_{\mathrm{g}} \Delta \mathrm{L} / \lambda+\Phi_{\mathrm{m}}\right] / 2\right)$

In Fig. (c), $\mathrm{k}_{\mathrm{e}}=1-4 \mathrm{k}(1-\mathrm{k}) \cos ^{2}\left(\Phi_{\mathrm{m}} / 2\right)$

Hence ke $=1$ can be achieved by the proper choice of $\Phi \mathrm{m}$.

\section{CONCLUSION}

There are number of techniques to compensate the chromatic dispersion of an optical signal travelling along the optical fiber. The dispersion compensation using digital filters is the most effective way of compensating it. It is a new class of digital filters implemented in the optical domain called all pass filters. All pass filters are lossless filters which offer the flexibility to tune a desired phase response arbitrarily close by increasing the number of stages keeping magnitude response of a system unchanged. The fully tunable all pass filters having $100 \mathrm{GHz}$ FSR and negligible polarization dependence have been fabricated with tuning range of $+100 \mathrm{ps} / \mathrm{nm}$, a pass band width of 50 $\mathrm{GHz}$ and group delay ripple of $<3$-ps peak are demonstrated. With the careful design of APF's together with the feedback equalization used at the receiver, the $10 \mathrm{Gbps}$ WDM system with FSR $=50 \mathrm{GHz}, \mathrm{OSNR}=22.7$ at BER of 10- 9 may be realized.

\section{REFERENCES}

[1] C.H. Cheng, "Signal Processing for Optical Communication," IEEE Signal Processing Magazine, vol. 23, no. 1, pp. 88-96, 2006.

[2] G. Keiser, "Optical Fiber Communication," McGrawHill International series, Third Edition, 2000.

[3] C.D. Poole, J.M. Wiesenfeld, D.J. DiGiovanni, A.M. Vengsarkar, "Optical fiber-based dispersion compensation using higher order modes near cutoff," Journal of Lightwave Technology, vol. 12, no. 10, pp. 1746-1758, 1994.

[4] N.M. litchinitser, D.B. Patterson, "Analysis of Fiber Bragg Gratings for Dispersion Compensation in Reflective and Transmissive Geometries," Journal of Lightwave Technology, vol. 15, no. 8, pp. 1303-1313, 1997.

[5] D. Pastor, J. Capmany, D. Ortega, V. Tatay, and J. Marti, "Design of Apodized Linearly Chirped Fiber Gratings for Dispersion Compensation," Journal of Lightwave Technology, vol. 14, no. 11, pp. 25812588, 1996.

[6] P.M. Watts, V. Mikhailov, S. Savory, P. Bayvel, M. Glick, M. Lobel, B. Christinsin, P. Krikpatrick, S. Shange, and R.I. Killey, "Performance of Single-Mode Fibers Links using Electronic Feed-Forward and Decision Feedback Equalizers," IEEE Photonics Technology Letter, vol. 17, no. 10, pp. 2206-2208, 2005.
[7] S. Cao, J. Chen, J.N. Damask, C.R. Doerr, L. Guiziou, G. Harvey, Y. Hibino, H.Li, S. Suzuki, K.Y. Wu, and P. Xie, "Interleaver Technology: Comparisons and Applications Requirements," Journal of Lightwave Technology, vol. 22, no. 1, pp. 281-289, 2004.

[8] P.A. Regalia, S.K. Mitra, P.P. Vaidyanathan, "The Digital All Pass Filter: A versatile Signal Processing building Block," Proceedings of the IEEE, vol. 76, no. 1, pp. 19-37, 1988.

[9] S.K. Mitra, K. Hirano, "Digital All Pass Filters," IEEE transaction circuits and systems, vol. CAS 21, no. 9, pp. 688-700, 1974.

[10] en.wikipedia.org/wiki/Fiber-optic communication.

[11] J.M. Senior, "Optical Fiber Communications Principles and Practice" Harlow Pearson 2005 Prentice Hall International Series in optoelectronics", second edition, no. 3, 2005.

[12] G. Lenz and C.K. Madsen, "General Optical All-Pass Filter Structures for Dispersion Control in WDM Systems," Journal of Lightwave Technology, vol. 17, no. 7, pp. 1248-1254, 1999.

[13] C.K. Madsen and G. Lenz, "Optical All Pass Filters for Phase Response Design with Applications for Dispersion Compensation," IEEE Photonics Technology Letter, vol. 10, no. 7, pp. 994-996, 1998.

[14] C.K. Madsen, J.A. Walker, J.E. Ford, K.W. Goossen, T.N. Nielsen, and G. Lenz, "A Tunable Dispersion Compensating MEMS All-Pass Filter," IEEE Photonics Technology Letter, vol. 12, no. 6, pp. 651653,2000

[15] C.K. Madsen, G. Lenz, A.J. Bruce, M.A. Cappuzzo, L.T. Gomez, and R.E. Scotti et al., "Integrated All Pass Filters for Tunable Dispersion and Dispersion Slope Compensation," IEEE Photonics Technology Letter, vol. 11, no. 12, pp. 1623-1625, 1999. 